\title{
Early cicatricial lagophthalmos release with pentagonal wedge resection of the scar, fat redistribution, and full-thickness skin grafting
}

\author{
Chan Min Chung ${ }^{1}$, \\ Seung Wan Tak ${ }^{1}$, \\ Hyoseob Lim ${ }^{1}$, \\ Sang-Hun Cho ${ }^{1}$, \\ Jong Wook Lee ${ }^{2}$ \\ ${ }^{1}$ Department of Plastic and \\ Reconstructive Surgery, Hallym \\ University Sacred Heart Hospital, \\ Hallym University College of Medicine, \\ Anyang; ${ }^{2}$ Department of Plastic and \\ Reconstructive Surgery, Hallym \\ University Hangang Sacred Heart \\ Hospital, Seoul, Korea
}

\begin{abstract}
The specialized structure of the upper eyelid ensures complete closure of the eye and eyeball sealing. An upper eyelid injury can cause various symptoms associated with eyeball trauma, not just scar formation or eyelid deformity. In this report, we describe a case of lagophthalmos observed after wound repair in a patient with a crushing injury caused by a grinder. Several surgical techniques are used to treat lagophthalmos or scar contracture. In most cases, a releasing procedure is performed after 6 months of initial repair. However, if the patient has severe symptoms that are not relieved by conservative care, early revision is inevitable. We describe a case of early lagophthalmos successfully resolved with pentagonal wedge resection, fat redistribution, and full-thickness skin grafting. After the revisional surgery, we observed that the patient regained the ability to completely close the injured eyelid, with restoration of function and favorable cosmetic outcomes. Pentagonal wedge resection to release a retracted structure, fat redistribution to prevent readhesion, and full-thickness skin grafting for enough amount of skin to regain upper eyelid function is useful for scar release and lagophthalmos following crushing injuries of the upper eyelid.
\end{abstract}

Keywords: Contracture / Eye movements / Eyelids / Skin transplantation

\section{INTRODUCTION}

The eyelids perform several important functions, primarily protecting the anterior eyeball from injury and maintaining the tear film integrity over the anterior surface of the globe [1,2]. Furthermore, eyelid movements (e.g., raising or tightening the eyelids) are associated with expression of emotions such as fear, disgust, or surprise, and lubricate the eyeball to prevent keratitis [1]. Crushing injuries of the eyelids can cause eyelid movement disorders in addition to significant scarring. Scar contractures of the eyelids affect eyelid functions, and in cases of severe con-

\footnotetext{
Correspondence: Hyoseob Lim

Department of Plastic and Reconstructive Surgery, Hallym University Sacred Heart Hospital, Hallym University College of Medicine, 22 Gwanpyeong-ro

170beon-gil, Dongan-gu, Anyang 14068, Korea

E-mail: hyoseob.lim@gmail.com

Received October 3, 2019 / Revised October 31, 2019 / Accepted November 4, 2019
}

tractures, local flap techniques, including Z- or W-plasty, may not achieve adequate scar release. Skin grafting should be considered in patients with lack of adequate eyelid skin. We describe a case with successful scar revision using the pentagonal wedge resection technique, fat redistribution, and skin grafting that showed excellent postoperative functional and cosmetic outcomes.

\section{CASE REPORT}

A 62-year-old man with a vertical crushing injury in his left facial area caused by a grinder visited our emergency department. His left upper eyelid was cut and showed a partial levator aponeurotic rupture (Fig. 1). Debridement and wound closure were performed with a levator aponeurotic repair the day after the onset of trauma. The wound healing was unremarkable. 

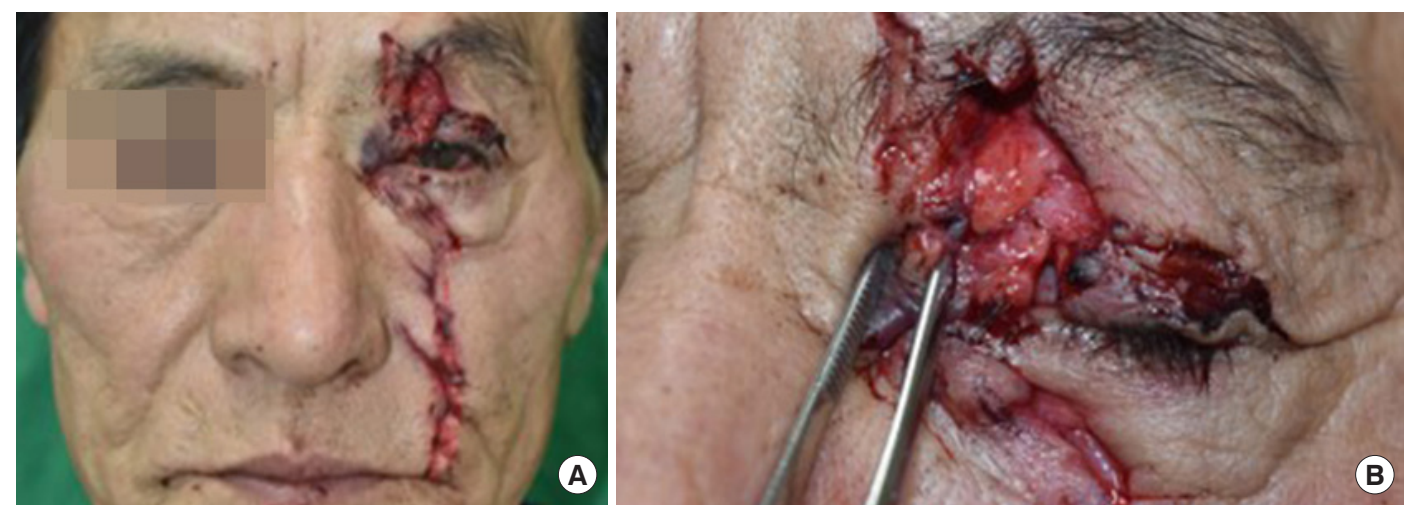

Fig. 1. Photographs showing the initial wound following a grinder injury. (A) Entire face. (B) Cut to the upper eyelid with tarsal plate and levator aponeurosis rupture.
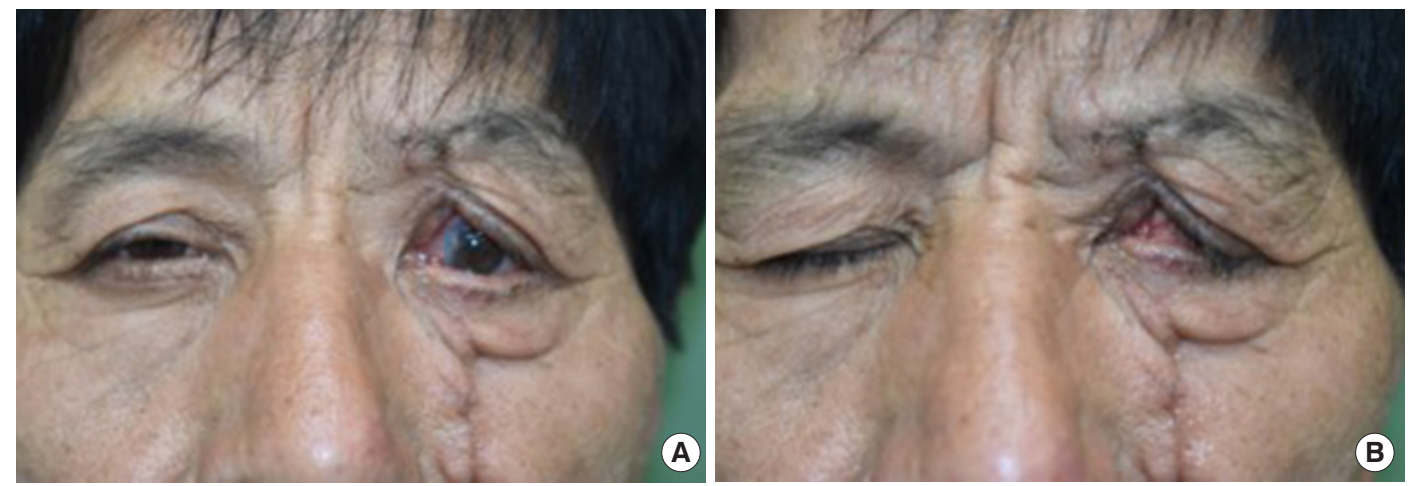

Fig. 2. Photographs obtained 45 days postoperatively showing lagophthalmos. (A) Eyes are open. (B) Eyes are shut.
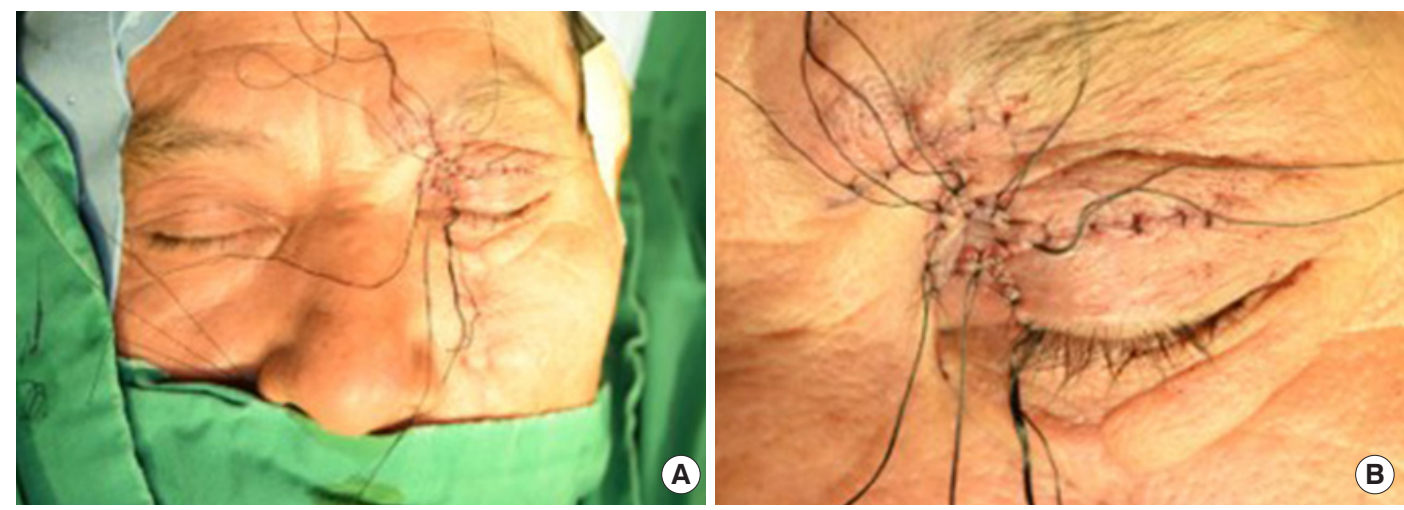

Fig. 3. Intraoperative photographs showing full-thickness skin grafting. (A) Entire face. (B) A magnified view of the left upper eyelid.

Approximately 45 days later, he developed a severe upper eyelid scar contracture that resulted in lagophthalmos, with exposure of $5 \mathrm{~mm}$ of his left eyeball when both eyes were fully closed (Fig. 2). As the patient was also followed up by the ophthalmology department, keratitis was identified right after its initial development and had been treated conservatively. However, his compliance to the ophthalmic conservative treatments was poor because the therapeutic contact lens kept dropping out of his eye continuously owing to the severe lagophthalmos. Hence, early lagophthalmos release was inevitable, and scar release with pentagonal wedge resection, fat redistribution, and fullthickness skin grafting (FTSG) were performed under general anesthesia. Pentagonal wedge resection was performed, and the scar tissue was dissected just anterior to the tarsus. After scar release, we observed a skin defect measuring $3 \times 1 \mathrm{~cm}$ in size. The eyelid margin was approximated first with minimal tension and without any eyelid deformity, followed by repair of the conjunctiva. Then, subcutaneous fat tissue redistribution was 

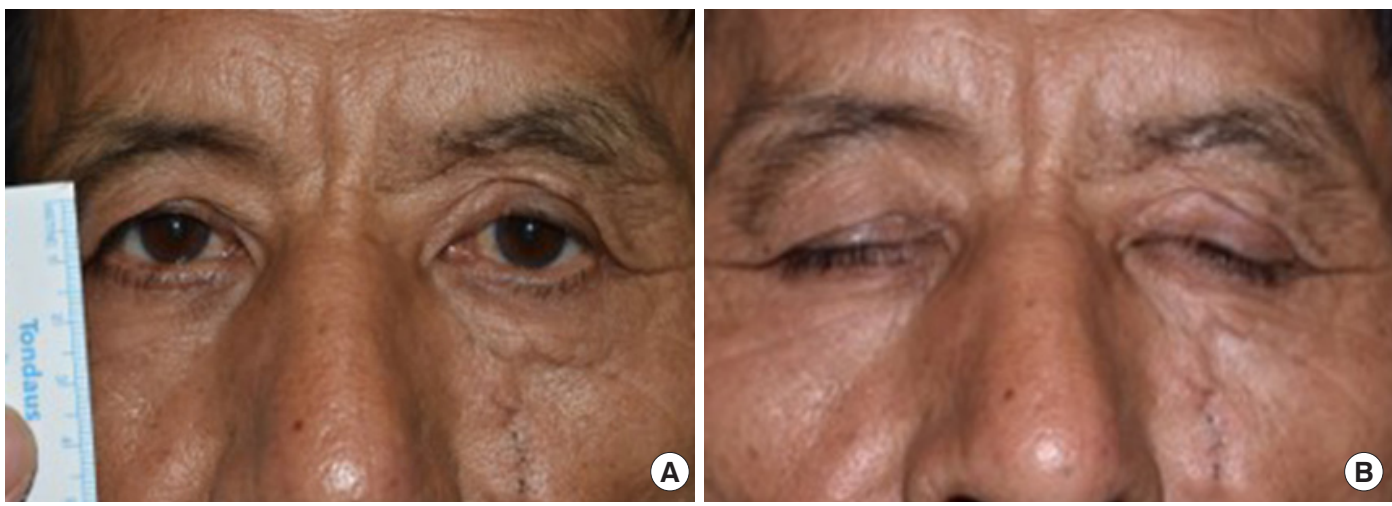

Fig. 4. Photographs obtained 18 months postoperatively showing full upper eyelid closure. (A) Eyes are open. (B) Eyes are shut.

performed to prevent readhesion of the thin upper eyelid skin or orbicularis oculi muscle to the levator complex at an undesirable level, and to prepare the nutritional bed for skin tissue. The aponeurotic fat was advanced and anchored on the tarsal plate and carefully handled to cover the whole levator complex. Then, the skin flaps around the redistributed fat were mobilized to achieve maximal coverage of the pentagonally excised defect. Skin flaps on the medial (glabella) and lateral sides were advanced for bilateral coverage using 5-0 PDS and 6-0 nylon sutures. After the advancement flap placement, a defect measuring $1.5 \times 1.0 \mathrm{~cm}$ persisted and required skin grafting. Fullthickness skin $(2.0 \times 1.0 \mathrm{~cm})$ harvested from the left posterior auricular area was defatted and grafted onto the upper eyelid and fixed with 6-0 nylon sutures with a tie-over dressing using 6-0 silk to secure the graft (Fig. 3). At the 10-day follow-up, we observed an excellent postoperative outcome, and he was able to fully close his upper eyelid. He had regained his ability to completely close the injured eyelid at the 18-month postoperative follow-up (Fig. 4), and no readhesion has been observed since then.

\section{DISCUSSION}

The eyelid plays a key role in maintaining the integrity of the corneal surface and in lubricating and protecting the surface of the eyeball $[3,4]$. Lagophthalmos, which results in upper eyelid dysfunction, can cause dry eyes, which predispose the patient to infection and can even lead to loss of vision. Therefore, eyelid deformity repair is primarily aimed at complete coverage of the eyeball and preservation of levator function [1,4].

Several reconstruction techniques have been used to repair upper eyelid defects, including local flaps and skin or dermofat grafting. Morley et al. [5] and Espinoza and Prost [3] have established protocols for upper eyelid reconstruction depending on the involvement of the tarsal plate, lacrimal canaliculi, and adjacent structures.

The present case shows the successful outcome of scar release with pentagonal wedge resection, fat redistribution, and FTSG. For successful releasing of upper eyelid scar contracture resulting in lagophthalmos, several crucial procedures are required, including the following: (1) complete excision or release of the contracted scar tissue; (2) preventive method from readhesion; and (3) reconstruction technique for skin defect due to previous procedures. In this case, pentagonal wedge resection of the scar was performed to release the retracted structure and to get a reapproximation of the eyelid margins [6]. Furthermore, by pentagonal wedge resection, the original lid height, which was shortened by cicatricial lagophthalmos, was recovered. A subsequent preaponeurotic fat redistribution to advance the preaponeurotic fat to the tarsal plate was adopted to prevent readhesion of the thin upper eyelid skin or orbicularis oculi muscle to the levator aponeurosis. This technique has been addressed by Lee et al. [7] to treat upper eyelid retraction after cosmetic eyelid surgery. The final step was skin grafting on the skin defect area with the posterior auricular skin, which mimics the upper eyelid skin in the aspect of softness and color. As the first step of the revision surgery, pentagonal excision, included skin excision, additional skin was needed in the final step.

FTSG offers the following advantages over the other upper eyelid coverage methods. First, the metabolic requirements of the grafted full-thickness skin are low, and the upper eyelid receives an abundant vascular supply for optimal graft survival [8]. Second, it is associated with low donor site morbidity. Third, late postoperative complications, including hypertrophy or contracture, are easily manageable with the application of anti-cicatricial silicone materials or finger massage [9]. Considering the aforementioned advantages, FTSG was performed to cover the defect that occurred secondary to the pentagonal wedge resection in this case. The posterior auricular skin is one of the best donor sites for upper eyelid skin defect coverage and 
was used as the donor site in our case owing to the following advantages: (1) the eyelid skin is the thinnest on the body. The thinness of the posterior auricular skin matches that of the eyelid skin; (2) a posterior auricular skin graft offers an excellent color match compared with non-facial donor sites; and (3) this area is hairless, the least sun damaged, and less noticeable [10].

As the production of collagen and scar remodeling continues approximately 6 months after injury, revisional operation is usually performed after half a year from the initial surgery [11]. As the scar contracture is most severe during 6 to 8 weeks after the onset of trauma, many upper eyelid revision surgeries occur after this period and mostly at least 6 months after the initial surgery. However, early lagophthalmos release was performed after approximately 1.5 months from the primary repair in this case because of the progressing keratitis in spite of the conservative therapies. A favorable outcome could be obtained in this case with early revision and proper surgical procedures. Along with surgical interventions, postoperative cicatricial sequelae can be prevented and reversed using massage, steroid injections, and silicone gel application, which should be continued for at least 6 months postoperatively.

In conclusion, we report an educational case of a severe lagophthalmos successfully released with pentagonal wedge resection, fat redistribution, and FTSG. During the healing period after the initial repair, lagophthalmos can present with severe ophthalmic symptoms. If ophthalmic problems could not be relieved even with vigorous conservative care, early revision before complete scar maturation could be a treatment option. The case showed the cosmetically and functionally desirable outcomes with early revision of contracted scar tissue with proper scar release, prevention of readhesion with inner tissue and fat position control, and the addition of a sufficient amount of skin.

\section{NOTES}

\section{Conflict of interest}

No potential conflict of interest relevant to this article was reported.

\section{Ethical approval}

The study was approved by the Institutional Review Board of Hallym University Sacred Heart Hospital (IRB No. 2019-09017) and performed in accordance with the principles of the Declaration of Helsinki. Written informed consent was obtained.

\section{Patient consent}

The patient provided written informed consent for the publication and the use of his images.

\section{ORCID}

Chan Min Chung https://orcid.org/0000-0002-2755-9440

Seung Wan Tak https://orcid.org/0000-0001-7744-5559

Hyoseob Lim https://orcid.org/0000-0002-6251-8281

Sang-Hun Cho https://orcid.org/0000-0001-5906-9828

Jong Wook Lee https://orcid.org/0000-0002-3244-949X

\section{REFERENCES}

1. Rucker JC. Normal and abnormal lid function. Handb Clin Neurol 2011;102:403-24.

2. Chiummariello S, Calzoni C, Pica A, Desgro MD, Alfano C. Reconstruction of the traumatic eyelid injuries: a 6-years experience. Ann Ital Chir 2013;84:73-6.

3. Espinoza GM, Prost AM. Upper eyelid reconstruction. Facial Plast Surg Clin North Am 2016;24:173-82.

4. Yesiloglu N, Sirinoglu H, Sarici M, Temiz G, Guvercin E. A simple method for the treatment of cicatricial ectropion and eyelid contraction in patients with periocular burn: vertical V-Y advancement of the eyelid. Burns 2014;40:1820-1.

5. Morley AM, deSousa JL, Selva D, Malhotra R. Techniques of upper eyelid reconstruction. Surv Ophthalmol 2010;55:256-71.

6. Vrcek I, Chou E, Blaydon S, Shore J. Wingtip flap for reconstruction of full-thickness upper and lower eyelid defects. Ophthalmic Plast Reconstr Surg 2017;33:144-6.

7. Lee JH, Kim YD, Woo KI, Johnson ON 3rd. Preaponeurotic fat advancement in levator recession for treatment of upper eyelid retraction. Plast Reconstr Surg 2015;135:680e-690e.

8. Zlatarova ZI, Nenkova BN, Softova EB. Eyelid reconstruction with full thickness skin grafts after carcinoma excision. Folia Med (Plovdiv) 2016;58:42-7.

9. Rathore DS, Chickadasarahilli S, Crossman R, Mehta P, Ahluwalia HS. Full thickness skin grafts in periocular reconstructions: long-term outcomes. Ophthalmic Plast Reconstr Surg 2014;30:517-20.

10. Shorr N, Goldberg RA, McCann JD, Hoenig JA, Li TG. Upper eyelid skin grafting: an effective treatment for lagophthalmos following blepharoplasty. Plast Reconstr Surg 2003;112:1444-8.

11. Son D, Harijan A. Overview of surgical scar prevention and management. J Korean Med Sci 2014;29:751-7. 